\title{
DESEMPENHO DOS MODELOS CLIGEN, LARS-WG E PGECLIMA_R NA SIMULAÇÃO DE SÉRIES DIÁRIAS DE TEMPERATURA MÁXIMA DO AR PARA LOCALIDADES DO ESTADO DO PARANÁ
}

\section{JORIM S. DAS VIRGENS FILHO ${ }^{1}$, RAFAEL B. DE OLIVEIRA ${ }^{2}$, MAYSA DE L. LEITE ${ }^{3}$, RODRIGO Y. TSUKAHARA ${ }^{4}$}

\begin{abstract}
RESUMO: Este trabalho teve o objetivo de avaliar o desempenho dos modelos CLIGEN, LARSWG e PGECLIMA_R na simulação de séries diárias de temperatura máxima do ar para localidades do Estado do Paraná. Foram utilizadas séries históricas de temperatura máxima do ar das localidades de Campo Mourão, Castro, Curitiba, Ivaí, Londrina, Maringá e Paranaguá. Foram geradas cinco séries de temperatura máxima do ar para cada modelo, nas localidades avaliadas, que foram confrontadas com as respectivas séries históricas. O processo de validação foi composto de análises estatísticas através de testes de significância (sobre as médias mensais (teste t), sobre a variância das médias mensais (teste F), sobre a forma das distribuições de frequência (teste K-S)), de gráficos de tendência anual, de tabelas com os índices " $r$ " de Pearson, "d" de Willmott e "c" de Camargo-Sentelhas. Na simulação de temperatura máxima do ar, os modelos PGECLIMA_R e LARS-WG não diferiram em desempenho, obtendo poucas rejeições e bons resultados nos índices "c", "d" e "r". No entanto, o CLIGEN obteve resultado abaixo do esperado, superestimando as temperaturas máximas do ar em dias úmidos e, subestimando-as em dias secos, nas localidades avaliadas.
\end{abstract}

PALAVRAS-CHAVE: simuladores climáticos, estatística climatológica, clima do Paraná, séries climáticas sintéticas.

\section{PERFORMANCE OF THE MODELS CLIGEN, LARS-WG AND PGECLIMA_R IN SIMULATION OF DAILY SERIES OF MAXIMUM AIR TEMPERATURE FOR LOCALITIES IN THE STATE OF PARANA, BRAZIL}

\begin{abstract}
This study aimed to evaluate the performance of the models CLIGEN, LARS-WG and PGECLIMA_R in the simulation of daily series of maximum air temperature for localities in the state of Parana, Brazil. It was used time series of maximum air temperature in the localities of Campo Mourão, Castro, Curitiba, Ivaí, Londrina, Maringa and Paranaguá. The validation process consisted of statistical analysis using significance tests (on the monthly average ( $\mathrm{t}$ test), on the variance of monthly average ( $\mathrm{F}$ test), on the shape of frequency distributions (K-S test)), of annual trend graphics, of tables with the " $r$ " index of Pearson, "d" index of Willmott and "c" index of Camargo-Sentelhas. In the simulation of maximum air temperature the PGECLIMA_R and LARSWG generators were not different in performance, obtaining fewer rejections and good results for the "c", "d" and "r" indexes. However CLIGEN obtained the worst results, overestimating the maximum temperatures on wet days and underestimating them on dry days in the localities evaluated in the state of Parana.
\end{abstract}

KEYWORDS: weather generators, climatological statistics, Parana climate, synthetic climate series.

\footnotetext{
${ }^{1}$ Matemático Estatístico, Doutor em Agronomia, Prof. Associado do Departamento de Matemática e Estatística, Universidade Estadual de Ponta Grossa - UEPG, Ponta Grossa - PR, jvirgens@uepg.br.

${ }^{2}$ Acadêmico do curso de Engenharia de Computação, Universidade Estadual de Ponta Grossa - UEPG, Ponta Grossa - PR, rafa.boliveira@hotmail.com.

${ }^{3}$ Eng $^{\underline{a}}$ Agrônoma, Doutora em Agronomia, Prof. Associado, Departamento de Biologia Geral, Universidade Estadual de Ponta Grossa, Ponta Grossa - PR, mleite@uepg.br.

${ }^{4}$ Eng ${ }^{\mathrm{O}}$ Agrônomo, Doutorando em Agronomia, Coordenador de Pesquisa em Agrometeorologia, Fundação ABC Pesquisa e Desenvolvimento Agropecuário, Castro - PR, rodrigo@ fundacaoabc.org.br.

Recebido pelo Conselho Editorial em: 3-1-2012

Aprovado pelo Conselho Editorial em: 30-1-2013
} 


\section{INTRODUÇÃO}

O crescimento, o desenvolvimento e a produtividade de uma cultura agrícola são afetados pela variabilidade climática, por intermédio de respostas lineares e não lineares aos elementos climáticos. Assim, conforme STRECK \& ALBERTO (2006), possíveis mudanças no clima podem aumentar o risco do agronegócio, uma vez que os efeitos negativos desta possibilidade têm maior impacto em países de economia frágil e não consolidada que compreendem a maioria dos países do bloco econômico em desenvolvimento, visto que, nestes países, os subsídios agrícolas são menores, e os agricultores, menos capitalizados.

De acordo com MELLO et al. (2008), os aumentos na temperatura do ar podem causar aumento da evaporação à superfície, provocando alterações no balanço hídrico da vegetação natural e das culturas agrícolas. Esse efeito deve ser mais negativo em regiões onde predominam a agricultura de sequeiro, a não ser que o aumento de temperatura do ar seja acompanhado de aumento e/ou regularização no regime de chuvas.

Nas atividades agronômicas, a temperatura máxima do ar exerce influência bioclimática ao longo do ano: nos meses frios, pela sua ação desvernalizante, e, nos meses quentes, pela sua ação deprimente, tanto no crescimento (tamanho) como no desenvolvimento (estádio) das plantas. A vernalização é um processo de acumulação de baixas temperaturas por parte da planta desde o estádio de semente germinada até o momento de mudança de fase vegetativa para reprodutiva, o que é necessário à sua produção. Este efeito pode ser suprimido pela ação de temperaturas elevadas durante vários dias. Por outro lado, as altas temperaturas nos meses quentes deprimem o crescimento e aceleram o desenvolvimento das plantas, podendo diminuir a fotossíntese, a respiração e a produção de matéria seca (SILVA et al., 2005).

Em estudos realizados sobre o desenvolvimento de batata cultivar Asterix, em Santa Maria-RS, sob condições de alterações climáticas, STRECK et al. (2006) observaram que o aumento de $2{ }^{\circ} \mathrm{C}$ na temperatura do ar foi suficiente para modificar a duração de algumas fases de desenvolvimento da cultura. KRISTENSEN et al. (2011), avaliando a resposta do rendimento do trigo de inverno sob um regime de mudanças climáticas, na Dinamarca, verificaram que um aumento da temperatura do ar no verão provoca baixos rendimentos na produção. Por outro lado, no inverno, o aumento da temperatura pode proporcionar alta produtividade da cultura.

Dados climáticos históricos são importantes para todas as aplicações envolvendo modelagem de agroecossistemas, porém, muitas vezes, os mesmos não estão disponíveis ou os registros referem-se a períodos curtos. Assim, nestes casos, geradores de dados climáticos podem tornar-se opção oportuna, por gerarem sinteticamente séries diárias para as diversas variáveis climáticas, que servirão como entrada para os modelos agroecossistêmicos. Vários geradores de dados climáticos, como o CLIGEN (NICKS et al., 1995), LARS-WG (SEMENOV \& BARROW, 1997), PGECLIMA_R (VIRGENS FILHO et al., 2010a; VIRGENS FILHO et al., 2010b) entre outros, são citados na literatura como ferramentas eficientes na simulação de cenários climáticos futuros para avaliação de modelos agronômicos ou hidrológicos, tendo em vista possíveis mudanças climáticas.

GUO et al. (2010), utilizando dados climáticos gerados pelo CLIGEN, avaliaram a resposta do rendimento de culturas agrícolas e a eficiência do uso da água sob alterações do clima para o norte da China e concluíram, baseados num dos cenários climáticos simulados, que o trigo em regime de aquecimento atmosférico aumentou seu rendimento médio. Todavia, neste mesmo cenário, a cultura do milho apresentou decréscimo em sua produção, fazendo com que os autores observassem que altas temperaturas não apenas afetam o rendimento agrícola, como também têm um efeito positivo sobre o uso eficiente da água, principalmente no que se refere à intensificação da evapotranspiração.

Utilizando o gerador LARS-WG, MORIONDO et al. (2010) avaliaram a sustentabilidade agrícola sob alterações climáticas, na região da Toscana (Itália), e concluíram que o setor agrícola é estruturalmente vulnerável a estas mudanças do clima, uma vez que as variações de temperatura e a 
ocorrência de eventos extremos na precipitação pluvial afetam a produtividade agrícola e as variáveis ambientais, o que sugere o desenvolvimento de estratégias adaptativas para a mitigação dos efeitos climáticos.

Com o intuito de simular cenários climáticos baseados em possíveis alterações climáticas para região fitogeográfica dos Campos Gerais - PR, VIRGENS FILHO \& LEITE (2010) aplicaram o PGECLIMA_R na simulação de cenários para as variáveis climáticas precipitação pluvial e temperatura do ar, baseados na projeção climática para 2100, publicada no terceiro relatório de avaliação do IPCC - Painel Intergovernamental sobre Mudanças Climáticas (IPCC, 2001). Os autores concluíram que, no melhor cenário, onde se projetou aumento médio de $1,4{ }^{\circ} \mathrm{C}$ na temperatura média e $14 \%$ de incremento na precipitação pluvial, poderá haver melhor desenvolvimento da floresta de araucária em decorrência de melhora significativa da atividade cambial. Todavia, para um cenário com aumento de $5,8^{\circ} \mathrm{C}$ da temperatura média do ar e $58 \%$ de acréscimo da quantidade de precipitação, poderão ocorrer eventos extremos diários para esses elementos climáticos, que influenciarão negativamente na hidratação e na desidratação das árvores, que, como consequência principal, apresentarão variações no crescimento diamétrico durante as $24 \mathrm{~h}$ do dia.

Percebe-se, assim, que os geradores de dados climáticos promovem uma interação de natureza probabilística do clima com componentes de outros sistemas de natureza agronômica e/ou hidrológica, que fornecem uma metodologia para medir o risco de incerteza climática que está relacionada com gerenciamentos alternativos em empreendimentos com agroecossistemas. Sendo assim, conforme preconiza ZANETTI et al. (2006), torna-se necessário que esses dados simulados passem por uma avaliação, a fim de analisar sua confiabilidade e a representação das condições climáticas reais do local de interesse. Em outras palavras, significa ter a garantia de que as propriedades estatísticas contidas nas séries históricas de cada variável climática foram preservadas.

ABABAEI et al. (2010) avaliaram o LARS-WG para 65 locais, no Irã, e observaram que o desempenho do gerador para reproduzir as médias mensais foi muito melhor do que para reproduzir as variâncias mensais. Além disso, recomendaram fortemente que cada local fosse avaliado distintamente antes da utilização do gerador, uma vez que para as variáveis temperatura máxima e temperatura mínima, a reprodução dos desvios-padrão mensais não foi satisfatória. Segundo MAVROMATIS \& HANSEN (2001), este é um problema de viés estatístico que ocorre frequentemente com os geradores de dados climáticos, que tendem a não representar satisfatoriamente a variabilidade interanual das séries climáticas geradas.

Haja vista a importância dos geradores de dados climáticos nos processos decisórios relacionados ao gerenciamento de agroecossistemas e o fato de que a temperatura do ar é uma variável climática determinante no crescimento e no desenvolvimento das plantas. Este trabalho objetivou avaliar o desempenho dos modelos CLIGEN, LARS-WG e PGECLIMA_R na simulação de séries diárias de temperatura máxima do ar para localidades do Estado do Paraná.

\section{MATERIAL E MÉTODOS}

Esta pesquisa foi desenvolvida junto ao Laboratório de Estatística Computacional Aplicada, pertencente ao Departamento de Matemática e Estatística da Universidade Estadual de Ponta Grossa. Para tanto, foram selecionadas sete localidades do Estado do Paraná a partir de dados de estações meteorológicas convencionais, disponibilizados pelo Instituto Nacional de Meteorologia (INMET), localidades (Tabela 1) que devem expressar a diversidade climática para a temperatura máxima do ar no Estado.

As séries de dados utilizadas neste trabalho abrangeram 48 anos de dados históricos (1961 2008). Destes, 25 anos (1961 - 1985) foram usados para a parametrização dos simuladores e 23 anos (1986 - 2008) foram utilizados para a comparação estatística das séries históricas e simuladas. 
TABELA 1. Localização geográfica das estações meteorológicas. Geographical location of weather stations, State of Parana, Brazil.

\begin{tabular}{cccc}
\hline Localidade & Lat. (S) & Long. (W) & Alt. (m) \\
\hline Campo Mourão (L1) & $24^{\circ} 03^{\prime}$ & $52^{\circ} 22^{\prime}$ & 616 \\
Castro (L2) & $24^{\circ} 47^{\prime}$ & $50^{\circ} 00^{\prime}$ & 1008 \\
Curitiba (L3) & $25^{\circ} 26^{\prime}$ & $49^{\circ} 16^{\prime}$ & 923 \\
Ivaí (L4) & $25^{\circ} 00^{\prime}$ & $50^{\circ} 51^{\prime}$ & 808 \\
Londrina (L5) & $23^{\circ} 19^{\prime}$ & $51^{\circ} 08^{\prime}$ & 566 \\
Maringá (L6) & $23^{\circ} 24^{\prime}$ & $51^{\circ} 55^{\prime}$ & 542 \\
Paranaguá (L7) & $25^{\circ} 32^{\prime}$ & $48^{\circ} 31^{\prime}$ & 4 \\
\hline
\end{tabular}

Na maioria das vezes, os simuladores de dados climáticos assumem a distribuição normal dos dados diários de temperatura do ar (tanto para a temperatura mínima como para a máxima), no processo de parametrização dos modelos para a simulação dos dados. Os três simuladores climáticos avaliados nesta pesquisa (CLIGEN, LARS-WG e PGECLIMA_R) trabalham de forma similar no processo de simulação dos dados de temperatura do ar. Inicialmente, simulam a ocorrência de precipitação pluvial por processos estocásticos. Caso seja determinada uma condição de ocorrência de precipitação pluvial para o dia em questão, o mesmo será considerado um dia úmido, e serão gerados valores para a temperatura mínima e máxima do ar para um dia com alguma quantidade de precipitação pluvial. Em caso contrário, o dia será considerado como seco, e serão gerados valores para as temperaturas mínima e máxima do ar, para um dia sem ocorrência de precipitação pluviométrica. O PGECLIMA_R e o CLIGEN utilizam a cadeia de Markov de dois estados para a decisão de ocorrência ou não de precipitação, enquanto no LARS-WG a simulação de ocorrência de precipitação é modelada por um modelo exponencial misto, onde são gerados distintamente dias úmidos e secos.

Para a comparação e validação dos dados simulados em relação aos dados históricos, foram geradas cinco séries de dados sintéticos de temperatura máxima do ar, para cada um dos simuladores e localidades, sendo que, em todos os casos, foram utilizados os mesmos períodos ( 25 anos) para a parametrização dos modelos e simulados 23 anos de dados. Após o processo de simulação, confrontaram-se as séries de dados simulados com as históricas que não foram utilizadas na parametrização, gerando assim uma independência estatística entre os dados.

No processo de comparação dos dados simulados, utilizou-se uma planilha eletrônica (BrOffice-Calc), onde os dados foram separados mensalmente em dias úmidos e secos, que foram analisados estatisticamente por meio do teste $t$ para a comparação das médias mensais, de teste $\mathrm{F}$ para análise da variabilidade mensal e do teste Kolmogorov-Smirnov (K-S) para analisar as distribuições dos dados, assumindo, em todos os casos, o nível de significância de 5\%. Para complementar a análise de desempenho dos três simuladores climáticos, foram elaborados gráficos de tendência dos valores médios mensais e tabelas com os valores do coeficiente de correlação "r" de Pearson, do índice de concordância "d" de Willmott e do coeficiente de desempenho "c" de Camargo-Sentelhas utilizados em GHERARDI et al. (2008), com finalidade semelhante.

\section{RESULTADOS E DISCUSSÃO}

Observa-se, na Tabela 2, onde é computada a quantidade de séries simuladas reprovadas para cada localidade e modelo em cada um dos testes para a temperatura máxima do ar em dias úmidos, que, para o teste $t$, os simuladores PGECLIMA_R e LARS-WG obtiveram resultados aproximados, em que o LARSWG foi reprovado em 70 testes, o que representa 16,67\% do total de 420 testes (cinco simulações para sete localidades, nos doze meses do ano) e o PGECLIMA_R em 78 (18,57\%) testes, representando um bom desempenho na reprodução das médias. Por outro lado, o CLIGEN não obteve resultado satisfatório, com $273(65 \%)$ testes rejeitados. Para o teste F, o LARS-WG obteve o melhor resultado, com $102(24,29 \%)$ testes rejeitados, enquanto PGECLIMA_R e o CLIGEN obtiveram 180 (42,86\%) e 194 (46,19\%) testes rejeitados, respectivamente, o que significa que os mesmos apresentaram desempenho ruim na recriação da variabilidade interanual das médias, o que, segundo MAVROMATIS \& HANSEN (2001), constitui-se em dos problemas dos geradores de clima. No teste K-S, o PGECLIMA_R mostrou-se melhor, com 113 (26,9\%) testes rejeitados, enquanto o LARS-WG obteve $142(33,81 \%)$ rejeições. O CLIGEN apresentou fraco desempenho no teste K-S com 299 (71,19\%) testes rejeitados, significando que o mesmo não conseguiu reproduzir de maneira aceitável a distribuição frequencial dos dados de temperatura máxima do ar em dias com ocorrência de precipitação pluvial. 
TABELA 2. Resultado da contagem dos testes estatísticos rejeitados para a temperatura máxima do ar em dias úmidos. Results of statistical tests rejected for maximum air temperature on wet days, State of Parana, Brazil.

\begin{tabular}{|c|c|c|c|c|c|c|c|c|c|}
\hline & \multicolumn{3}{|c|}{ Teste $\mathrm{t}(5 \%)$} & \multicolumn{3}{|c|}{ Teste F $(5 \%)$} & \multicolumn{3}{|c|}{ Teste K-S (5\%) } \\
\hline & \multicolumn{9}{|c|}{ CLIGEN LARS-WG PGECLIMA_R CLIGEN LARS-WG PGECLIMA_R CLIGEN LARS-WG PGECLIMA_R } \\
\hline L1 & 48 & 13 & 12 & 19 & 16 & 26 & 56 & 24 & 11 \\
\hline L2 & 29 & 12 & 17 & 49 & 19 & 37 & 27 & 27 & 30 \\
\hline L3 & 23 & 7 & 0 & 27 & 5 & 19 & 24 & 22 & 5 \\
\hline L4 & 45 & 11 & 15 & 21 & 16 & 23 & 41 & 18 & 17 \\
\hline L5 & 38 & 12 & 14 & 27 & 17 & 29 & 42 & 24 & 25 \\
\hline L6 & 50 & 14 & 19 & 22 & 17 & 19 & 60 & 17 & 12 \\
\hline L7 & 40 & 1 & 1 & 29 & 12 & 27 & 49 & 10 & 13 \\
\hline Total & 273 & 70 & 78 & 194 & 102 & 180 & 299 & 142 & 113 \\
\hline
\end{tabular}

Na Figura 1, onde é possível visualizar a tendência anual das médias da temperatura máxima do ar em dias úmidos, verifica-se que o CLIGEN tende a superestimar quase todos os meses do ano, em todas as localidades, em torno de $1,5^{\circ} \mathrm{C}$ a $2{ }^{\circ} \mathrm{C}$, fato que, no mundo real, pode gerar eventos extremos previstos por MELLO et al. (2008), bem como os encontrados em STRECK et al. (2006) e KRISTENSEN et al. (2011), que prejudicam a produção agrícola e que também podem aumentar a mortalidade animal e vegetal do ambiente. No entanto, os simuladores LARS-WG e o PGECLIMA_R obtiveram resultados muito próximos das médias históricas, produzindo pequenas oscilações sobre a tendência anual.

Na Tabela 3, mostra-se a quantidade de testes rejeitados para cada um dos modelos na simulação de temperatura máxima do ar em dias secos, sendo que, para o teste $t$, os simuladores LARS-WG e PGECLIMA_R alcançaram bons resultados na reprodução das médias históricas, em que o PGECLIMA_R apresentou 91 (21,67\%) rejeições e o LARS-WG 99 (23,57\%) rejeições. O CLIGEN obteve um desempenho ruim, com 278 resultados rejeitados, que representa 66,20\% do total de testes. Para o teste F, o LARS-WG obteve o melhor resultado, com $134(31,90 \%)$ rejeições. Por outro lado, o CLIGEN e o PGECLIMA_R obtiveram resultados muito aquém do esperado, com $293(69,76 \%)$ e $319(75,95 \%)$ resultados rejeitados, respectivamente, o que significa que os mesmos reproduziram insatisfatoriamente a variabilidade interanual das médias mensais. No teste K-S, o PGECLIMA_R obteve o melhor resultado, com 204 (48,57\%) testes rejeitados, enquanto o LARS-WG e o CLIGEN obtiveram 239 (56,90\%) e $370(88,10 \%)$ rejeições, respectivamente, significando que os modelos apresentaram desempenhos mediano e muito ruim, respectivamente, na reprodução das distribuições de frequência da temperatura do ar em dias secos.

TABELA 3. Resultado da contagem dos testes estatísticos rejeitados para a temperatura máxima em dias secos. Results of statistical tests rejected for maximum air temperature on dry days, State of Parana, Brazil..

\begin{tabular}{|c|c|c|c|c|c|c|c|c|c|}
\hline & \multicolumn{3}{|c|}{ Teste $\mathrm{t}(5 \%)$} & \multicolumn{3}{|c|}{ Teste F $(5 \%)$} & \multicolumn{3}{|c|}{ Teste K-S (5\%) } \\
\hline & \multicolumn{9}{|c|}{ CLIGEN LARS-WG PGECLIMA_R CLIGEN LARS-WG PGECLIMA_R CLIGEN LARS-WG PGECLIMA_R } \\
\hline L1 & 32 & 21 & 15 & 43 & 15 & 48 & 51 & 37 & 26 \\
\hline L2 & 35 & 14 & 17 & 31 & 13 & 41 & 54 & 43 & 48 \\
\hline L3 & 49 & 13 & 0 & 40 & 7 & 33 & 57 & 36 & 7 \\
\hline L4 & 40 & 3 & 6 & 49 & 35 & 52 & 47 & 26 & 26 \\
\hline L5 & 35 & 20 & 16 & 48 & 24 & 56 & 55 & 48 & 44 \\
\hline L6 & 38 & 20 & 23 & 47 & 16 & 49 & 50 & 39 & 39 \\
\hline L7 & 49 & 8 & 14 & 35 & 24 & 40 & 56 & 10 & 14 \\
\hline Total & 278 & 99 & 91 & 293 & 134 & 319 & 370 & 239 & 204 \\
\hline
\end{tabular}



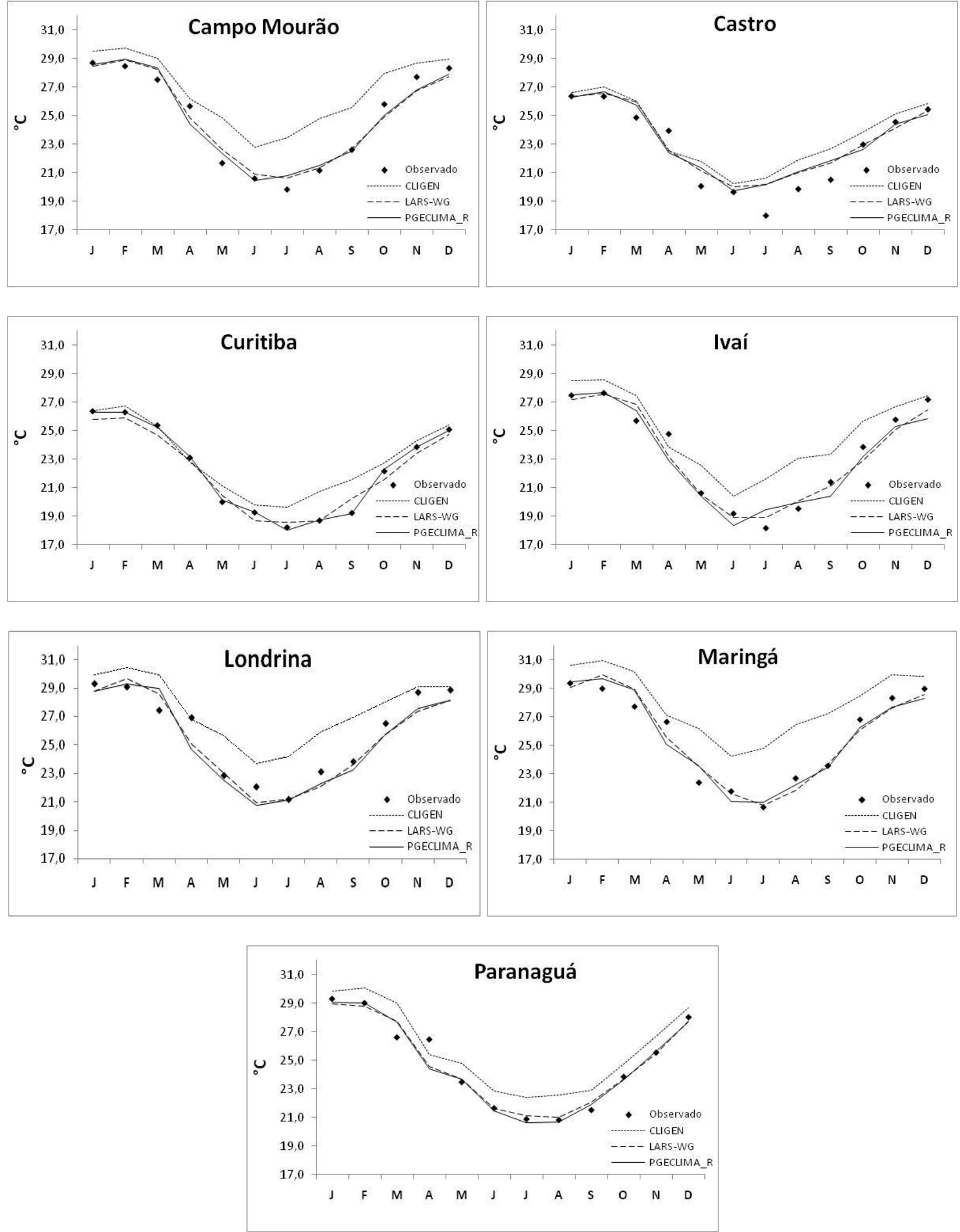

FIGURA 1. Tendência anual das séries de temperatura máxima do ar em dias úmidos, geradas pelos modelos CLIGEN, LARS-WG e PGECLIMA_R. Series annual trend of the maximum air temperature on wet days generated by CLIGEN, LARS-WG and PGECLIMA_R, State of Parana, Brazil. 
Na Figura 2, é possível verificar que o CLIGEN subestimou as médias históricas em aproximadamente $1{ }^{\circ} \mathrm{C}$ para todas as localidades, enquanto o PGECLIMA_R e o LARS-WG alcançaram resultados próximos às médias históricas, simulando bem as tendências anuais.
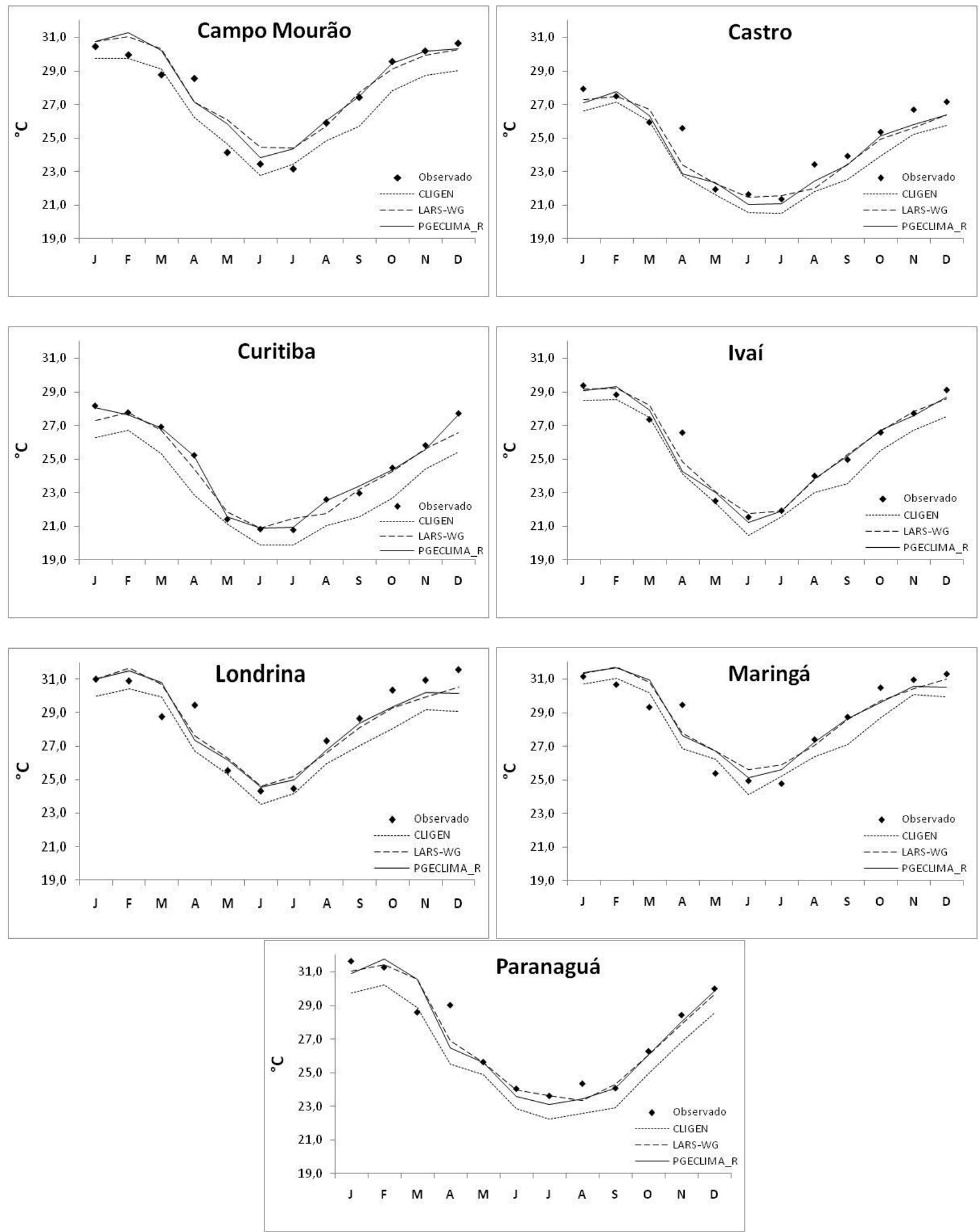

FIGURA 2. Tendência anual das séries de temperatura máxima do ar em dias secos, geradas pelos modelos CLIGEN, LARS-WG e PGECLIMA_R. Series annual trend of the maximum air temperature on dry days generated by CLIGEN, LARS-WG and PGECLIMA_R, State of Parana, Brazil. 
Pode-se observar, nas Tabelas 4 e 5, que o PGECLIMA_R apresentou certa vantagem na análise dos índices "c", "d" e "r" em relação aos outros dois modelos. De certa forma, estes índices mostram que os três geradores de clima apresentaram uma "forte correlação" na simulação das tendências históricas ao longo dos meses do ano; e, segundo o índice "c" de CAMARGO \& SENTELHAS (1997), na grande maioria das vezes, os mesmos alcançaram desempenhos considerados "muito bons" $(0,76$ a 0,85$)$, conforme escala do índice. É importante observar que isto não implica, necessariamente, representar bem as médias, os desvios-padrão e as distribuições de frequência dentro de cada mês, como ocorreu com o CLIGEN que, no conjunto de testes estatísticos, apresentou fraco desempenho.

TABELA 4. Índice de desempenho dos geradores avaliados na simulação de temperatura máxima do ar em dias úmidos. Performance index of the generators evaluated in the simulation of the maximum air temperature on wet days, State of Parana, Brazil.

\begin{tabular}{|c|c|c|c|c|c|c|c|c|c|}
\hline & \multicolumn{3}{|c|}{ Campo Mourão } & \multicolumn{3}{|c|}{ Castro } & \multicolumn{3}{|c|}{ Curitiba } \\
\hline & $\mathrm{r}$ & $\mathrm{d}$ & $\mathrm{c}$ & $\mathrm{r}$ & $\mathrm{d}$ & $\mathrm{c}$ & $\mathrm{r}$ & $\mathrm{d}$ & $\mathrm{c}$ \\
\hline CLIGEN & 0,8698 & 0,8691 & 0,7864 & 0,8764 & 0,9068 & 0,819 & 0,8801 & 0,8991 & 0,7913 \\
\hline LARS-WG & 0,8895 & 0,9284 & 0,8131 & 0,8737 & 0,9346 & 0,8103 & 0,8617 & 0,9189 & 0,7918 \\
\hline \multirow[t]{2}{*}{ PGECLIMA_R } & 0,8986 & 0,9370 & 0,8473 & 0,8972 & 0,9576 & 0,8356 & 0,9030 & 0,9473 & 0,8553 \\
\hline & $r$ & Ivaí & & \multicolumn{3}{|c|}{ Londrina } & \multicolumn{3}{|c|}{ Maringá } \\
\hline CLIGEN & $\frac{r}{0.8649}$ & $\frac{d}{0.8571}$ & $\frac{\mathrm{c}}{0.7413}$ & $\begin{array}{c}\mathrm{r} \\
0.8762\end{array}$ & $\frac{d}{0.9057}$ & $\frac{\mathrm{c}}{0.8035}$ & $\begin{array}{c}\mathrm{r} \\
0.8946\end{array}$ & $\frac{\mathrm{d}}{0.9011}$ & $\frac{\mathrm{c}}{0.8019}$ \\
\hline LARS-WG & 0,8777 & 0,9273 & 0,8139 & 0,8673 & 0,9237 & 0,8097 & 0,8754 & 0,9342 & 0,8234 \\
\hline \multirow[t]{3}{*}{ PGECLIMA_R } & 0,8914 & 0,9350 & 0,8335 & 0,8964 & 0,9412 & 0,8462 & 0,9038 & 0,9563 & 0,8643 \\
\hline & & & & \multicolumn{3}{|c|}{ Paranaguá } & & & \\
\hline & & & & $\mathrm{r}$ & $\mathrm{d}$ & $\mathrm{c}$ & & & \\
\hline CLIGEN & & & & 0,8961 & 0,9175 & 0,8123 & & & \\
\hline LARS-WG & & & & 0,8897 & 0,9481 & 0,8157 & & & \\
\hline PGECLIMA_R & & & & 0,9113 & 0,9643 & 0,8897 & & & \\
\hline
\end{tabular}

TABELA 5. Índice de desempenho dos geradores avaliados na simulação da temperatura máxima do ar em dias secos. Performance index of the generators evaluated in the simulation of the maximum air temperature on dry days, State of Parana, Brazil.

\begin{tabular}{|c|c|c|c|c|c|c|c|c|c|}
\hline & \multicolumn{3}{|c|}{ Campo Mourão } & \multicolumn{3}{|c|}{ Castro } & \multicolumn{3}{|c|}{ Curitiba } \\
\hline & $\mathrm{r}$ & $\mathrm{d}$ & $\mathrm{c}$ & $\mathrm{r}$ & $\mathrm{d}$ & $\mathrm{c}$ & $\mathrm{r}$ & $\mathrm{d}$ & $\mathrm{c}$ \\
\hline CLIGEN & 0,8591 & 0,9056 & 0,7815 & 0,8618 & 0,8946 & 0,7413 & 0,8434 & 0,8417 & 0,7099 \\
\hline LARS-WG & 0,8617 & 0,9227 & 0,7986 & 0,8709 & 0,9113 & 0,7916 & 0,8232 & 0,8906 & 0,7331 \\
\hline PGECLIMA_R & 0,8643 & $\begin{array}{c}0,9219 \\
\text { Ivaí }\end{array}$ & 0,8134 & 0,8712 & $\begin{array}{c}0,9198 \\
\text { Londrina }\end{array}$ & 0,7981 & 0,8577 & $\begin{array}{c}\text { 0,9184 } \\
\text { Maringá }\end{array}$ & 0,7877 \\
\hline & $\mathrm{r}$ & $\mathrm{d}$ & $\mathrm{c}$ & $\mathrm{r}$ & $\mathrm{d}$ & $\mathrm{c}$ & $\mathrm{r}$ & $\mathrm{d}$ & $\mathrm{c}$ \\
\hline CLIGEN & 0,8681 & 0,9007 & 0,7819 & 0,8431 & 0,8761 & 0,7531 & 0,8346 & 0,8864 & 0,7641 \\
\hline LARS-WG & 0,8769 & 0,9292 & 0,8148 & 0,8613 & 0,9178 & 0,8137 & 0,8589 & 0,8975 & 0,8197 \\
\hline PGECLIMA_R & 0,8696 & 0,9260 & 0,8052 & 0,8586 & $\begin{array}{c}0,9317 \\
\text { Paranaguá }\end{array}$ & 0,8317 & 0,8612 & 0,9013 & 0,8287 \\
\hline & & & & $\mathrm{r}$ & $\mathrm{d}$ & $\mathrm{c}$ & & & \\
\hline CLIGEN & & & & 0,8641 & 0,8617 & 0,7789 & & & \\
\hline LARS-WG & & & & 0,8746 & 0,9192 & 0,8065 & & & \\
\hline PGECLIMA_R & & & & 0,8894 & 0,9242 & 0,8130 & & & \\
\hline
\end{tabular}

\section{CONCLUSÕES}

Os modelos PGECLIMA_R e LARS-WG apresentam desempenhos equivalentes na simulação de séries diárias de temperatura máxima do ar, tanto em dias úmidos como em dias secos.

O modelo CLIGEN apresenta desempenho abaixo do esperado na simulação de séries diárias de temperatura máxima do ar.

Na simulação para dias úmidos, o CLIGEN superestima as temperaturas médias mensais históricas em até $2^{\circ} \mathrm{C}$, enquanto para dias secos subestima em aproximadamente $1{ }^{\circ} \mathrm{C}$. 


\section{AGRADECIMENTOS}

Ao CNPq, à Fundação Araucária do Paraná e ao FINEP, pelo suporte financeiro e concessão de bolsas de iniciação científica, bem como ao INMET, pela cessão das séries de dados.

\section{REFERÊNCIAS}

ABABAEI, B.; SOHRABI, T.; MIRZAEI, F.; KARIMI, B. Evaluation of a stochastic weather generator in different climates. Computer and Information Science, Toronto, v.3, n.3, p.217-229, 2010.

CAMARGO, A.P.; SENTELHAS, P.C. Avaliação do desempenho de diferentes métodos de estimativa da evapotranspiração potencial no Estado de São Paulo, Brasil. Revista Brasileira de Agrometeorologia, Santa Maria, v.5, p.89-97, 1997.

GHERARDI, B.; DOURADO NETO, D.; SENTELHAS, P.C. Caracterização espacial e temporal da temperatura média do ar, em escala diária e mensal, no Estado de Mato Grosso. Revista Brasileira de Agrometeorologia, Santa Maria, v.16, n.2, p.133-141, 2008.

GUO, R.; ZHONGHUI, L.; MO, X.; YANG, C. Responses of crop yield and water use efficiency to climate change in the North China Plain. Agricultural Water Management, Amsterdam, v.97, p.1.185-1.194, 2010.

IPCC. INTERGOVERNMENTAL PANEL ON CLIMATE CHANGE. Climate Change 2001: The Scientific Basis-Contribution of Working Group 1 to the IPCC Third Assessment Report. Cambridge: Cambridge Univ. Press, 2001. 17 p.

KRISTENSEN, K.; SCHELDE, K.; OLESEN, J. E. Winter wheat yield response to climate variability in Denmark. Journal of Agricultural Science, Nottingham, v.149, p.33-47, 2011.

MAVROMATIS, T.; HANSEN, J.W. Interannual variability characteristics and simulated crop response of four stochastic weather generators. Agricultural and Forest Meteorology, Amsterdam, v.109, p.283-296, 2001.

MELLO, E.L.; OLIVEIRA, F.A.; PRUSKI, F.F.; FIGUEIREDO, J.C. Efeito das mudanças climáticas na disponibilidade hídrica da bacia hidrográfica do Rio Paracatu. Engenharia Agrícola, Jaboticabal, v.28, n.4, p.635-644, 2008.

MORIONDO, M.; PACINI, C.; TROMBI, G.; VAZZANA, C.; BINDI, M. Sustainability of dairy farming system in Tuscany in a changing climate. European Journal of Agronomy, Amsterdam, v.32, p.80-90, 2010.

NICKS, A.D.; LANE, L.J.; GANDER, G.A. Weather generator: USDA-water erosion prediction project (WEPP). West Lafayette: USDA-ARS, 1995. cap.2, 22p.

SEMENOV, M.A.; BARROW, E.M. Use of a stochastic weather generator in the development of climate changes scenarios. Climatic Changes, Amsterdam, v.35, p.397-414, 1997.

SILVA, J.B.; LLOPART, M.P.; BOIASKI, N. Temperatura máxima do ar em Pelotas-RS - Tabelas de probabilidades em escalas de tempo pentadal. Revista Brasileira de Meteorologia, São José dos Campos, v.20, n.2, p.267-276, 2005.

STRECK, N.A.; ALBERTO, C.M. Simulação do impacto da mudança climática sobre a água disponível do solo em agroecossistemas de trigo, soja e milho, em Santa Maria - RS. Ciência Rural, Santa Maria, v.36, n.2, p.424-433, 2006.

STRECK, N.A.; LAGO, I.; ALBERTO, C.M.; BISOGNIN, D.A. Simulação do desenvolvimento da batata cultivar Asterix em cinco cenários de mudanças climáticas, em Santa Maria - RS. Bragantia, Campinas, v.65, n.4, p.693-702, 2006. 
VIRGENS FILHO, J.S.; FÉLIX, R.P.; LEITE, M.L.; TSUKAHARA, R. Y. Desenvolvimento de uma ferramenta computacional para geração de cenários climáticos, baseada em processos estocásticos. I - Interface gerenciadora do banco de dados. In: CONGRESO LATINOAMERICANO Y DEL CARIBE DE INGENIERÍA AGRÍCOLA, 9., CONGRESSO BRASILEIRO DE ENGENHARIA AGRÍCOLA, 39., 2010. Vitória. Anais... Vitória: Associação Brasileira de Engenharia Agrícola, 2010a. 1 CD-ROM.

VIRGENS FILHO, J.S.; LEITE, M.L. Simulation of climate scenarios for the region of Campos Gerais, State of Paraná, Brazil. In: IUFRO Landscape Ecology Working Group International Conference, 2010, Bragança-Portugal. Proceedings... Bragança-Portugal: Instituto Politécnico de Bragança, 2010. p.364-369.

VIRGENS FILHO, J.S.; OLIVEIRA, P.M.; LEITE, M.L.; TSUKAHARA, R.Y. Desenvolvimento de uma ferramenta computacional para geração de cenários climáticos, baseada em processos estocásticos. II - Interface de validação dos dados simulados. In: CONGRESO LATINOAMERICANO Y DEL CARIBE DE INGENIERÍA AGRÍCOLA, 9., CONGRESSO BRASILEIRO DE ENGENHARIA AGRÍCOLA, 39., 2010. Vitória. Anais... Vitória: Associação Brasileira de Engenharia Agrícola, 2010b. 1 CD-ROM.

ZANETTI, S.S.; OLIVEIRA, V.P.S.; PRUSKI, F.F. Validação do modelo ClimaBR em relação ao número de dias chuvosos e à precipitação total diária. Engenharia Agrícola, Jaboticabal, v.26, n.1, p.96-102, 2006. 